\title{
Jejunostomy Feeding Tube Placement in Gastrectomy Procedures: A Systematic Review
}

\section{Khalil Bazzi ${ }^{1 *}$, John Lahoud1, Charbel Sandroussi2,3, Jerome Martin Laurence ${ }^{2,3}$, Sharon Carey4, David Yeo ${ }^{2,3}$}

\author{
${ }^{1}$ The University of Notre Dame Australia, Sydney, Australia \\ ${ }^{2}$ Department of Hepatobiliary and Upper Gastrointestinal Surgery, Royal Prince Alfred Hospital, Camperdown, Australia \\ ${ }^{3}$ Institute of Academic Surgery, Royal Prince Alfred Hospital, Camperdown, Australia \\ ${ }^{4}$ Department of Nutrition and Dietetics, Royal Prince Alfred Hospital, Camperdown, Australia \\ Email: ^Khalil.bazzi@Outlook.com
}

How to cite this paper: Bazzi, K., Lahoud, J., Sandroussi, C., Laurence, J.M., Carey, S. and Yeo, D. (2017) Jejunostomy Feeding Tube Placement in Gastrectomy Procedures: A Systematic Review. Open Journal of Gastroenterology, 7, 52-64.

https://doi.org/10.4236/ojgas.2017.72007

Received: December 18, 2016

Accepted: February 12, 2017

Published: February 15, 2017

Copyright $\odot 2017$ by authors and Scientific Research Publishing Inc. This work is licensed under the Creative Commons Attribution International License (CC BY 4.0).

http://creativecommons.org/licenses/by/4.0/

\begin{abstract}
Many nutritional interventions have been developed to improve nutritional outcomes following upper gastrointestinal surgery. The aim of this systematic review was to investigate whether or not the routine use of intraoperative jejunostomy feeding tubes in partial and total gastrectomy procedures is warranted when assessing complications and nutritional benefits such as improved chemotherapy tolerance. An electronic search of MEDLINE, Web of Science, Embase and CINAHL databases was performed to identify studies which reported complications and/or post-operative outcomes of patients who received an intraoperative jejunostomy feeding tube in gastrectomy procedures. Five articles met the inclusion criteria $(n=636)$ with four retrospective cohort studies and one RCT. Studies varied in regards to the complications and nutritional outcomes reported. Jejunostomy feeding tube insertion may carry a risk of increased infectious complications but appears to reduce patient post-operative weight-loss and may improve chemotherapy tolerance. Due to the lack of high-quality studies, it is unclear if the routine use of an intraoperative jejunostomy feeding tube is indicated for all patients undergoing gastrectomy procedures or only those at a high-risk of post-operative malnutrition. More comprehensive research is recommended, particularly on the usefulness of home enteral nutrition post-gastrectomy.
\end{abstract}

\section{Keywords}

Gastrectomy, Jejunostomy, Feeding Jejunostomy Tube, Enteral Nutrition, Gastric Cancer 


\section{Introduction}

Gastric cancer is the fourth most common cancer behind lung, breast and colorectal cancers, but is the second most common cause of death from cancer with 700,000 deaths annually [1]. Although almost two-thirds of cases occur in developing countries, it remains a common problem in developed nations. For example, Australia has an incident age-standardised rate of 8.5 per 100,000 and an age-standardised mortality rate of 4.5 per 100,000 [2]. The definitive treatment for gastric cancer is resection, which depending on the location of the lesion, would warrant either a partial or total gastrectomy [3]. The utilisation of perioperative chemotherapy in addition to surgery for adenocarcinoma has been shown to improve patient survival compared with surgery alone [4].

Given the disruption to the alimentary canal that these procedures cause, there is no surprise that postoperative recovery is affected significantly by the patients' nutritional status. Patients who have undergone gastric resection are susceptible to malnutrition due to higher metabolic requirements, reduced oral intake and symptoms such as early satiety and dumping [5] [6] [7]. They often experience difficulty meeting their nutritional requirements in the acute postsurgical phase and therefore, experience complications associated with poor nutritional status such as impaired wound healing, reduced immune function and ultimately increased postoperative mortality [8] [9]. Impaired nutritional status restricts a patient's ability to tolerate chemotherapy [10], clearly indicating a need to achieve satisfactory post-operative nutritional status to optimise patient outcomes.

Many nutritional interventions have been developed to improve nutritional outcomes following surgery such as the early introduction of oral intake, total parenteral nutrition (TPN), nasojejunal/nasoduodenal feeding, and jejunostomy tube feeding. Previous literature indicates that early enteral nutrition (EEN) is preferred over TPN following gastrectomy procedures [11] [12] [13] [14]. The effectiveness of postoperative EEN is attributed to its ability to maintain gastrointestinal tract integrity and enhance immunological function [15] [16] [17]. In addition, EEN as compared with TPN, is less costly, produces less infectious complications and is easier for nursing staff to administer [11] [12].

Jejunostomy feeding as a method of enteral nutritional support following surgical intervention has gradually gained wide acceptance since first being described by Busch in 1858 [18] and hence is often recommended in current guidelines [19] [20]. A jejunostomy feeding tube can be inserted intraoperatively at the time of resection. However, the utilisation of a jejunostomy feeding tube is not without complications such as tube leakages, tube site infections, and even tube-associated mortality [21]. The aim of the current systematic review is to investigate whether or not the routine use of an intraoperative feeding jejunostomy tube is warranted in adult patients undergoing total and partial gastrectomy. This will be determined by assessing post-operative patient outcomes and complications. 


\section{Materials and Methods}

\subsection{Information Sources and Search Strategy}

A systematic review of the literature was conducted on the $20^{\text {th }}$ of November 2016 via MEDLINE (1966 to November 2016), Web of Science (1980 to November 2016), Embase (1980 to November 2016) and CINAHL (1980 to November 2016) databases. The following search terms were used:

Term 1: "esophageal neoplasm" OR "stomach neoplasm" OR "gastrectomy" OR "esophagectomy",

AND

Term 2: "jejunostomy" OR "jejunal tube" OR "jejunal feeding tube" OR "j-tube".

Articles were filtered to include English texts and human studies only. Case reports were excluded.

Studies that addressed the use of jejunostomy feeding tubes intraoperatively during total and/or partial gastrectomy were reviewed and suitability assessed for inclusion within the systematic review. The reference lists of all included articles were reviewed to obtain any additional studies not found within the initial search.

\subsection{Eligibility Criteria}

Studies were eligible for inclusion if they included the use of intraoperative jejunostomy feeding tube following total and/or partial gastrectomy and reported complications and/or post-operative patient outcomes. Studies which included other feeding methods in addition to jejunostomy such as total parenteral nutrition were included. Articles that explored the use of a jejunostomy feeding tube insertion at laparoscopic staging or as a result of a complication were excluded. Studies were limited to an adult population.

\subsection{Quality Assessment and Data Extraction}

Two reviewers (KB and JL) independently appraised all suitable studies using a modified Heyland review tool on a scale of 0 - 11 [22]. The criteria used to assess methodological quality and scope for bias included grading the use and presence of: randomisation, blinding, intention to treat analysis, method of patient selection, whether or not the description of outcomes was defined and if there was a baseline comparison of groups. Where the reviewers scored an article differently, it was discussed until a consensus was reached. In situations where a consensus was not reached, it was referred to a third independent reviewer (SC) for a final decision. Articles which received a score of $<3$ were excluded. Levels of evidence were assigned as per the Centre of evidence-based medicine (CEBM) [23].

Data extraction was performed by two researchers (KB and JL). Extracted data included study design, patient characteristics, tube-related complications, overall complications, and post-operative nutritional outcomes. 


\subsection{Statistical Analysis}

Due to the heterogenous nature of the articles, meta-analysis was unable to be performed.

\section{Results}

An initial search identified 762 articles, after duplicates were removed, relating to the insertion of intraoperative jejunostomy tubes for gastrectomy patients (Figure 1). The titles were screened for appropriateness and 84 full text articles were assessed for eligibility. Five articles met the criteria for eligibility.

Of the final 5 studies included, only one was a randomised control trial, with the other four being retrospective cohort reviews. The lack of high-quality data is demonstrated with four of the five studies carrying a CEBM level evidence of 4 (Tables 1-3). Two studies investigated jejunostomy tube outcomes in both partial and total gastrectomy patients [24] [25] while two studies investigated outcomes in total gastrectomy patients only [11] [26]. The study of Sun et al. compiled large amounts of partial and total gastrectomy patient data from the American College of Surgeons National Surgical Quality Improvement Program (NSQIP) database however outcomes were not differentiated based on procedure [27].

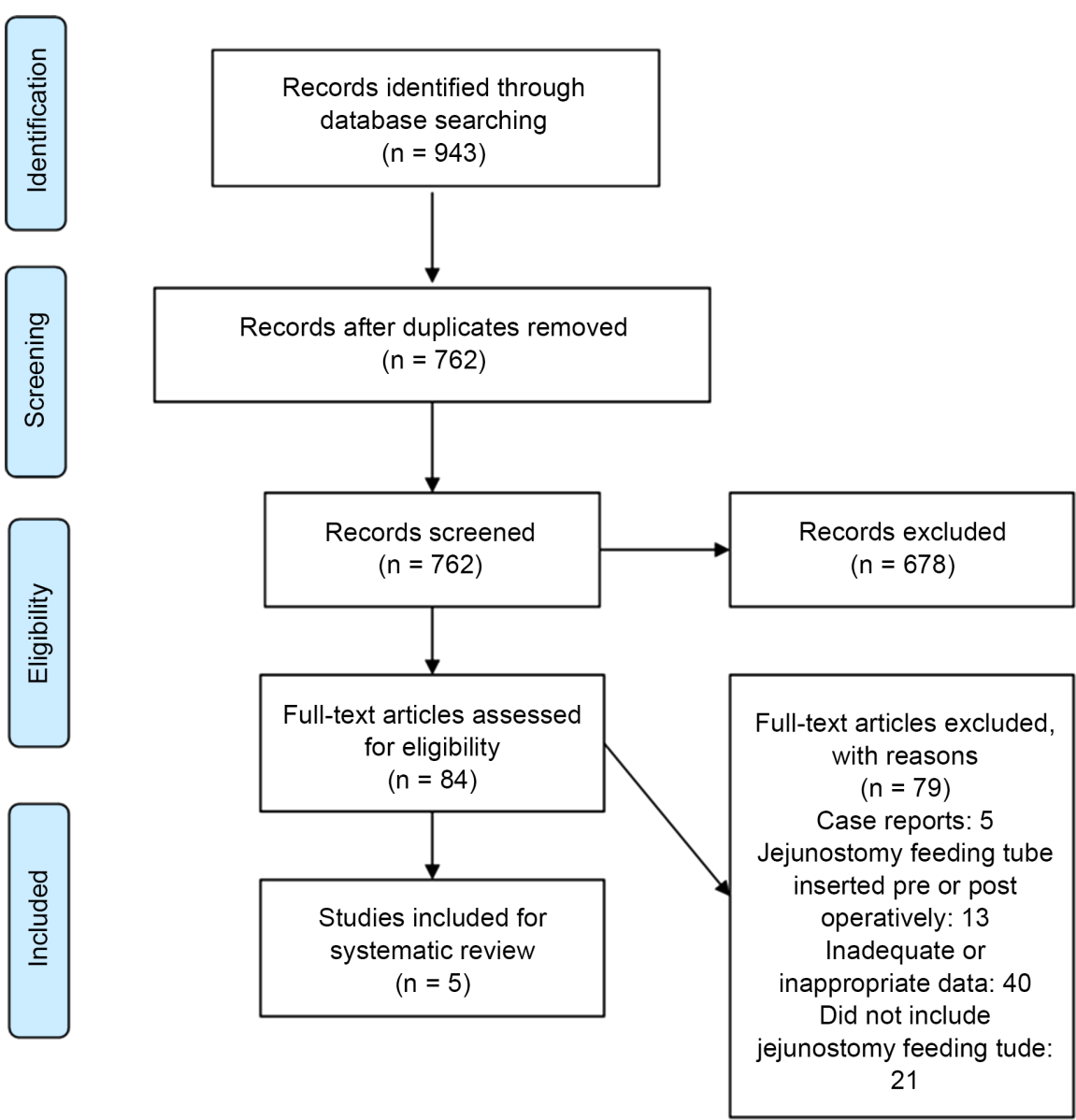

Figure 1. PRISMA. 
Table 1. Studies isolating total gastrectomy data.

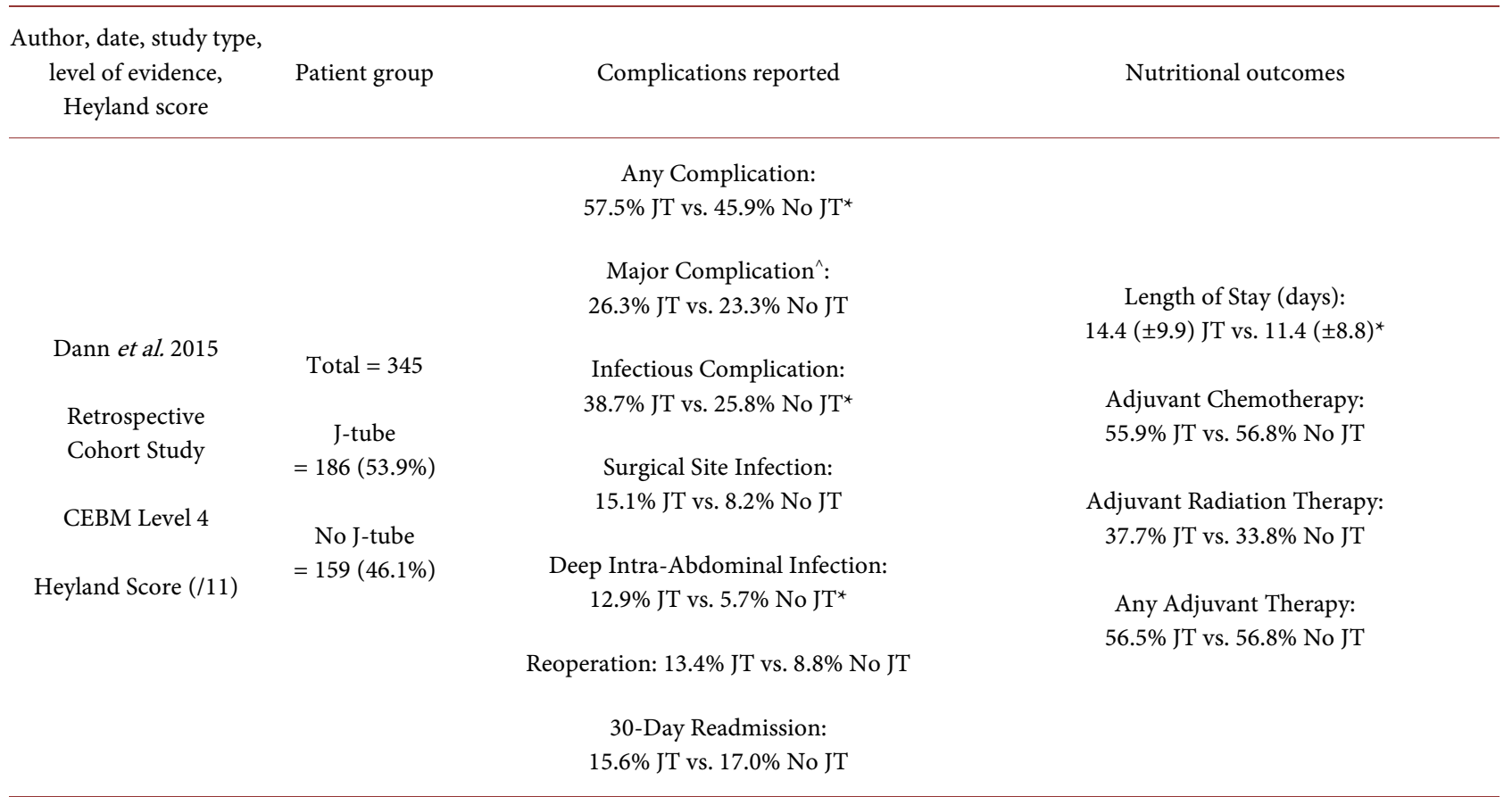

JT:

Heylen et al. 1987

Prospective

Randomised

Control Trial

CEBM Level 2

Heyland Score (/11): 5
Total $=20$

$\mathrm{J}$-tube $=10(50 \%)$

$\mathrm{TPN}=10(50 \%)$
Catheter Complications: 0\% Superficial Wound Abscess: 10\%

No Other Complications Reported

TPN:

Thrombosis: $10 \%$

Superficial Wound Abscess: 10\%

Deep Abscess: 10\%

No Other Complications Reported

Actual Calorie Intake vs. Prescribed Amount:

JT: $83 \%$ of Intended Target

TPN: Identical to Prescribed Amount

Total Caloric Intake ${ }^{* *}$ :

JT: $11,690 \mathrm{kcal} / \mathrm{pt} / 8$ days vs.

TPN: $12,200 \mathrm{kcal} / \mathrm{pt} / 8$ days

Weight Loss:

JT: $3.7 \mathrm{~kg}$ vs. TPN: $5.1 \mathrm{~kg}^{*}$

Mid-Upper Arm Circumference

JT: $-1.6 \mathrm{~cm}$ vs. TPN: $-0.4 \mathrm{~cm}$

Blood Biochemistry at Day $8^{* * *}$ :

No Significant Difference between JT and TPN

Any Complication:

$53 \%$ JT vs. $43 \%$ No JT

Infectious Complication:

$34 \%$ JT vs. $14 \%$ No JT

Patel et al. 2013

Total $=46$

Retrospective

Cohort Study

CEBM Level 4

Heyland Score (/11): 5

\section{J-tube: 32 (69.6\%)}

No J-tube:

$14(30.4 \%)$
Major Complication^^:

$6 \%$ JT vs. $0 \%$ No JT

Reoperation: $3 \%$ JT vs. $0 \%$ No JT

Bleeding Requiring Packed RBC Transfusion: $0 \% \mathrm{JT}$ and No JT

Anastomotic Leak: 0\% JT and 0\% No JT

30-Day Readmission: $22 \%$ JT vs. $21 \%$ No JT
Length of Stay (days):

$14(8-30)^{\mathrm{a}} \mathrm{JT}^{\mathrm{T}}$ vs. $10(5-15)^{\mathrm{a}}$ No JT*

Albumin Pre-Operative: $3.4(1.8-4.2)^{\mathrm{a}} \mathrm{JT}$ vs. $3.2(2.1-4.1)^{\mathrm{a}}$ No JT

Albumin 30-Day: $2.9(1.12-3.8)^{\mathrm{a}} \mathrm{JT}$ vs. $2.7(2.0-3.3)^{\mathrm{a}}$ No JT

Albumin 60-Day: $3.2(1.3-4.4)^{\mathrm{a}} \mathrm{JT}$ vs. $2.7(2.0-3.7)^{\mathrm{a}}$ No JT

Albumin 90-Day: $3.5(1.9-4.5)^{\mathrm{a}} \mathrm{JT}$ vs. $3.2(2.7-3.6)^{\mathrm{a}}$ No JT

Received Adjuvant Therapy Post-Op: $66 \%$ JT vs. $57 \%$ No JT 


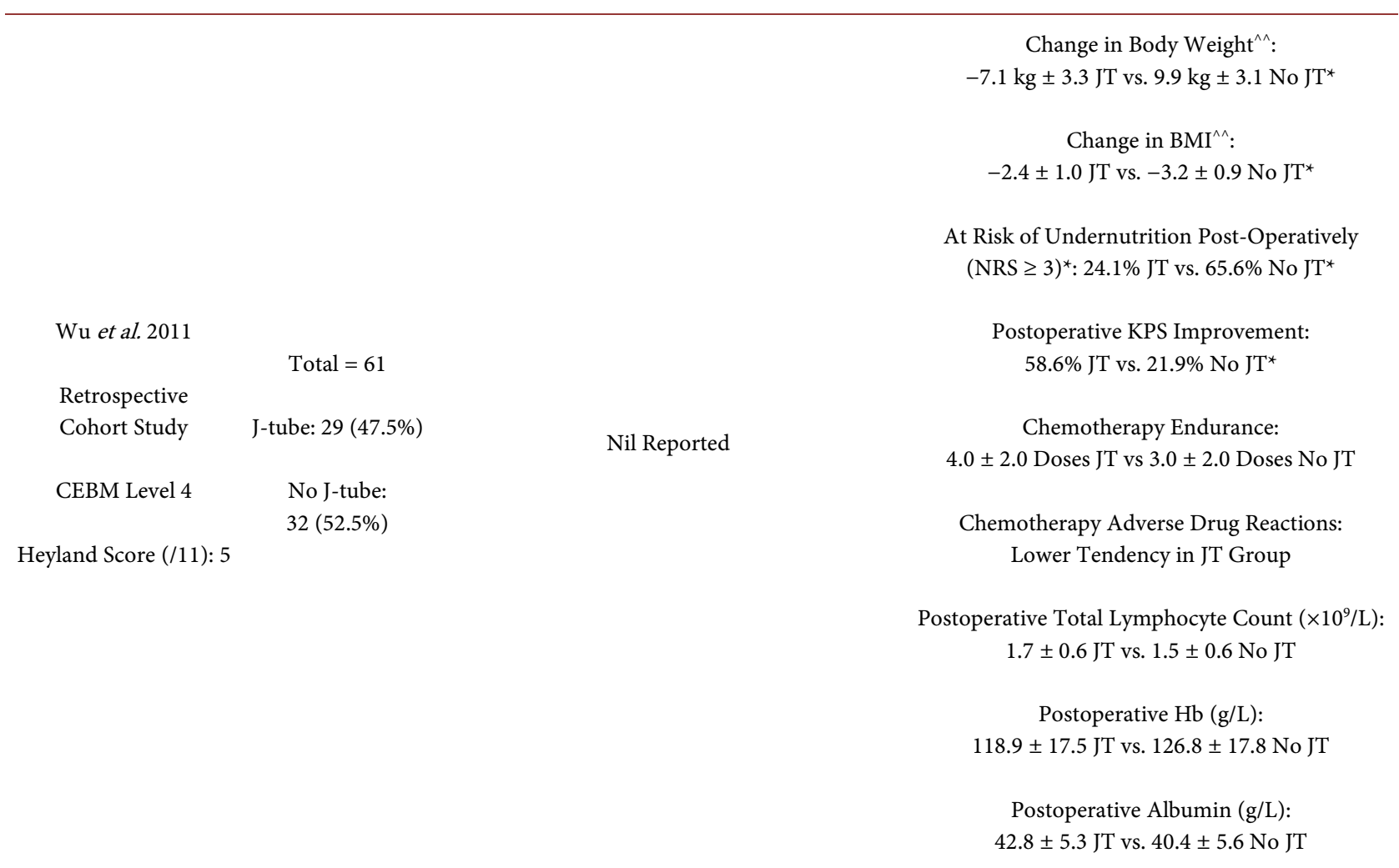

$\mathrm{JT}=$ Jejunostomy Tube Group; No JT = Jejunostomy Free Group; ${ }^{*}$ p-value $<0.05 ;{ }^{* *} 14 \%$ of global effective caloric support from peripheral venous infusion of glucose and/or sorbitol containing solutions; ${ }^{* * *}$ Blood biochemistry includes: albumin, transferring, thyroxin-binding pre-albumin, retinol-binding protein, liver function tests, haemoglobin, electrolyte determinations, total lymphocyte count; ${ }^{\wedge}$ Major complication $=$ Clavien Grade 3 - 5; ${ }^{\mathrm{a}}$ Mean (range); ${ }^{\wedge} 1$-week pre-op to 3 months post-op; NRS = Nutritional Risk Screening 2002; KPS = Karnofsky performance score; used to estimate patients' activities and quality of life.

\subsection{Total Gastrectomy Patients}

\subsubsection{Jejunostomy vs. No Jejunostomy}

Three retrospective cohort studies compared outcomes between total gastrectomy patients with and without a jejunostomy feeding tube (Table 1). Jejunostomy and jejunostomy-free patients of Dann et al. and Patel et al. were similar, however criteria for selection of jejunostomy tube insertion was not described [24] [25]. Dann et al. noted a statistically significant increase in any complication, infectious complications, and deep intra-abdominal infections in patients who received a jejunostomy tube [24]. These trends were also noted in the study of Patel et al:; however, the findings in that study were not statistically significant [25]. Both Patel and Dann also noted that jejunostomy tube placement was associated with increased infectious complications and overall postoperative complications respectively on multivariate regression analysis, independent of other variables such as TNM stage [24] [25]. The study of Wu et al. did not report on any complications [26].

Both Dann et al. and Patel et al. noted a statistically significant increase in length of hospital stay for patients with jejunostomy feeding tubes. Neither study noted any statistically significant differences between jejunostomy and jejunostomy-free patients regarding adjuvant therapy received [24] [25]. A trend 
towards higher albumin levels post-operatively in the jejunostomy feeding group was noted by Patel et al;; however, this was not statistically significant [25]. It is not clear when and if feeding through the jejunostomy tubes was initiated in the studies of Dann et al. and Patel et al., making it difficult to assess the benefits of post-operative enteral nutrition via jejunostomy tube in total gastrectomy patients in those studies [24] [25].

This contrasts with the study of $\mathrm{Wu}$ et al., where patients in the jejunostomy group were all fed from 72 hours postoperatively or onset of flatus. In addition, the jejunostomy group patients in $\mathrm{Wu}$ et al. underwent home enteral nutrition (HEN) for at least 3 months. Significant improvements in nutritional outcomes were noted when compared to jejunostomy free patients including reduced weight loss postoperatively, reduced risk of undernutrition postoperatively (as judged by the Nutritional Risk Screening 2002 tool), and postoperative improvement in Karnofsky performance score (an indicator of patient quality of life and activity). There was an increased tendency for jejunostomy patients to receive higher doses of chemotherapy and lower rates of adverse effects ( $p>$ 0.05). No significant differences were noted between patients at baseline [26].

\subsubsection{Jejunostomy vs. Total Parenteral Nutrition}

A small randomised control trial compared jejunostomy feeding with TPN in 20 patients following total gastrectomy (Table 1). Jejunostomy feeding resulted in no tube related complications. There was one case of thrombosis in the TPN group; however, it is unclear if this was a venous thrombosis related to the catheter. Results indicated comparable caloric intake between the two groups but there was significantly less weight loss in the jejunostomy feeding group $(3.7 \mathrm{~kg})$ compared with the TPN group (5.1 kg) when mean final body weights were compared $(p<0.01)[11]$. Baseline patient BMI and mean body weight were not described. There was a statistically significant difference between mid-upper arm circumferences post-operatively with a decrease in $1.6 \mathrm{~cm}$ noted in the jejunostomy group compared to a decrease of $0.4 \mathrm{~cm}$ in the TPN group. The authors hypothesised that the difference in postoperative mid-upper arm circumference were due to increased salt losses in the stools of jejunostomy patients. There were no statistically significant differences in blood biochemistry between the two groups post-operatively (Table 1). Heylen et al. did note that there was an increased frequency of diarrhoea and abdominal cramps in the jejunostomy group; however, this was alleviated in most cases by altering the rate of infusion and adding loperamide to the feed [11].

\subsection{Partial Gastrectomy Patients}

\section{Jejunostomy vs. No Jejunostomy}

Two retrospective cohort studies compared outcomes between partial gastrectomy patients with and without a jejunostomy feeding tube (Table 2). As in the data presented for total gastrectomy patients, Dann et al. and Patel et al. noted a statistically significant increase in infectious complications in jejunostomy patients [24] [25]. Dann et al. demonstrated an increase in surgical site wound 
Table 2. Studies isolating partial gastrectomy data.

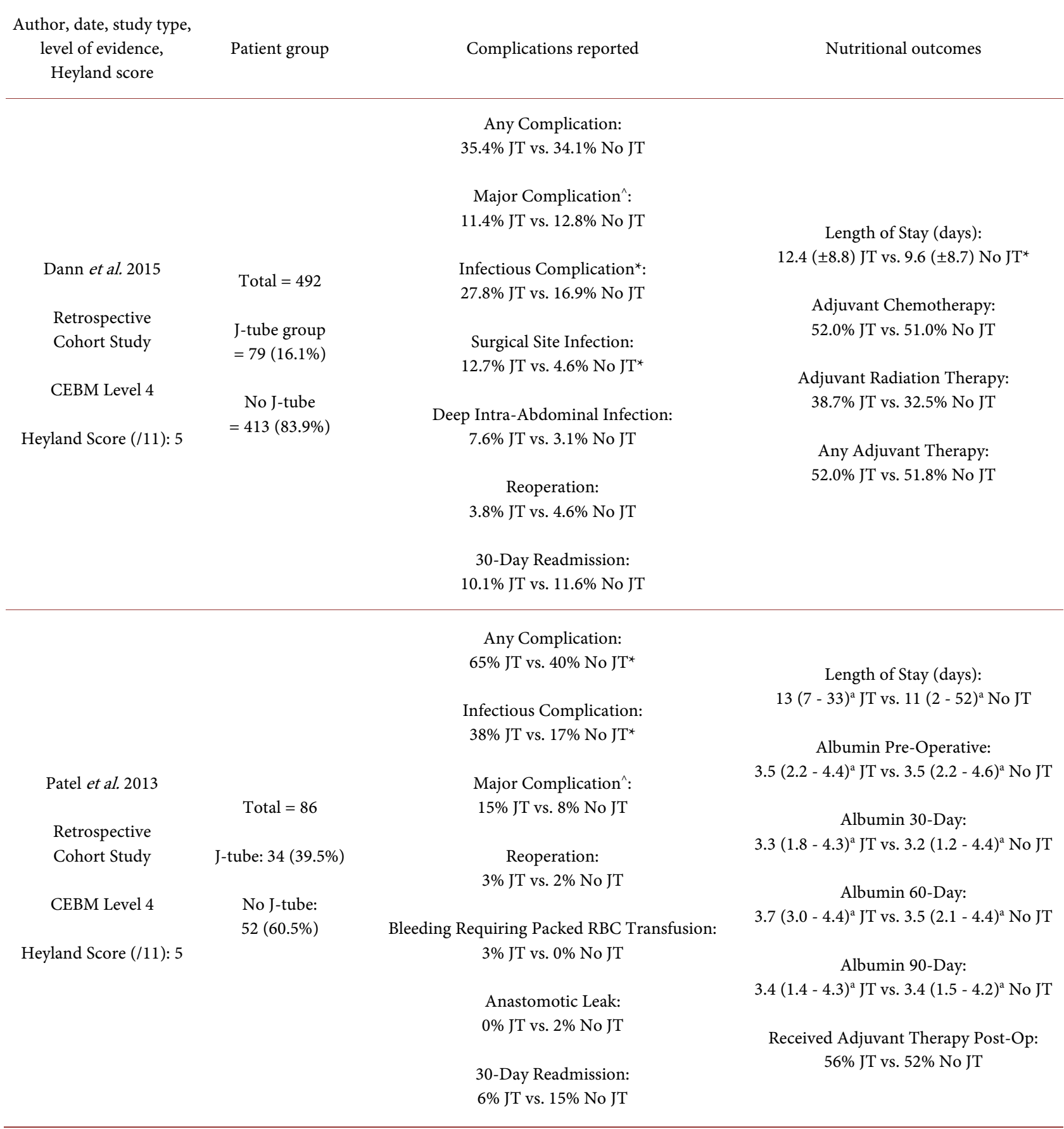

JT = Jejunostomy Tube Group; No JT = Jejunostomy Free Group; ${ }^{\star} \mathrm{p}$-value $<0.05 ;{ }^{\wedge}$ Major complication = Clavien Grade 3 - 5; ${ }^{\mathrm{a}}$ Mean (range).

infections in jejunostomy patients, which remained independently associated with jejunostomy tube insertion on multivariate analysis $(\mathrm{p}<0.05)$. Dann et al. found that jejunostomy patients had a longer hospital stay than those without a jejunostomy tube ( $\mathrm{p}<0.05$ ) [24] and neither Dann et al. nor Patel et al. noted a difference in adjuvant therapy received between groups [24] [25]. Once more, it was not explained how patients were chosen to receive jejunostomy feeding tubes and when or if they were delivered feeds through the tube. 


\subsection{Mixed Partial and Total Gastrectomy Data}

Jejunostomy vs. No Jejunostomy

Sun et al. utilised the largest, risk-adjusted, validated set of 30 day surgical outcomes in the United States to compare perioperative outcomes between gastrectomy patients who did or did not receive a jejunostomy feeding tube but the study did not differentiate outcomes based on the level of resection (i.e. partial vs. total gastrectomy) [27]. Of the 2980 patients, $71.4 \%$ underwent partial gastrectomy and $28.6 \%$ underwent total gastrectomy (Table 3). Only $24 \%$ of all patients received a jejunostomy tube, with the reasons for selection not described. Patients who received a jejunostomy tube were more likely to have recent weight loss, have undergone recent chemotherapy and radiotherapy, and more likely to have undergone total gastrectomy $(\mathrm{p}<0.05)$ [27]. These factors may demonstrate that surgeons typically reserve the insertion of a jejunostomy feeding tube to patients expected to suffer from postoperative malnutrition or suffer from more advanced disease. However, Sun et al. state that after adjustment with propensity matching the groups were highly similar. Aside from a slightly longer operative time for jejunostomy patients, the authors noted no statistically significant differences in mortality, overall complications, or any of the secondary outcomes. Jejunostomy group patients did experience higher rates of urinary tract infections $6.4 \%$ to $3.4 \%(\mathrm{p}<0.05)$ [27]. The authors postulated that a potential longer duration of catheterisation to monitor fluid balance may have been

Table 3. Mixed partial and total gastrectomy data.

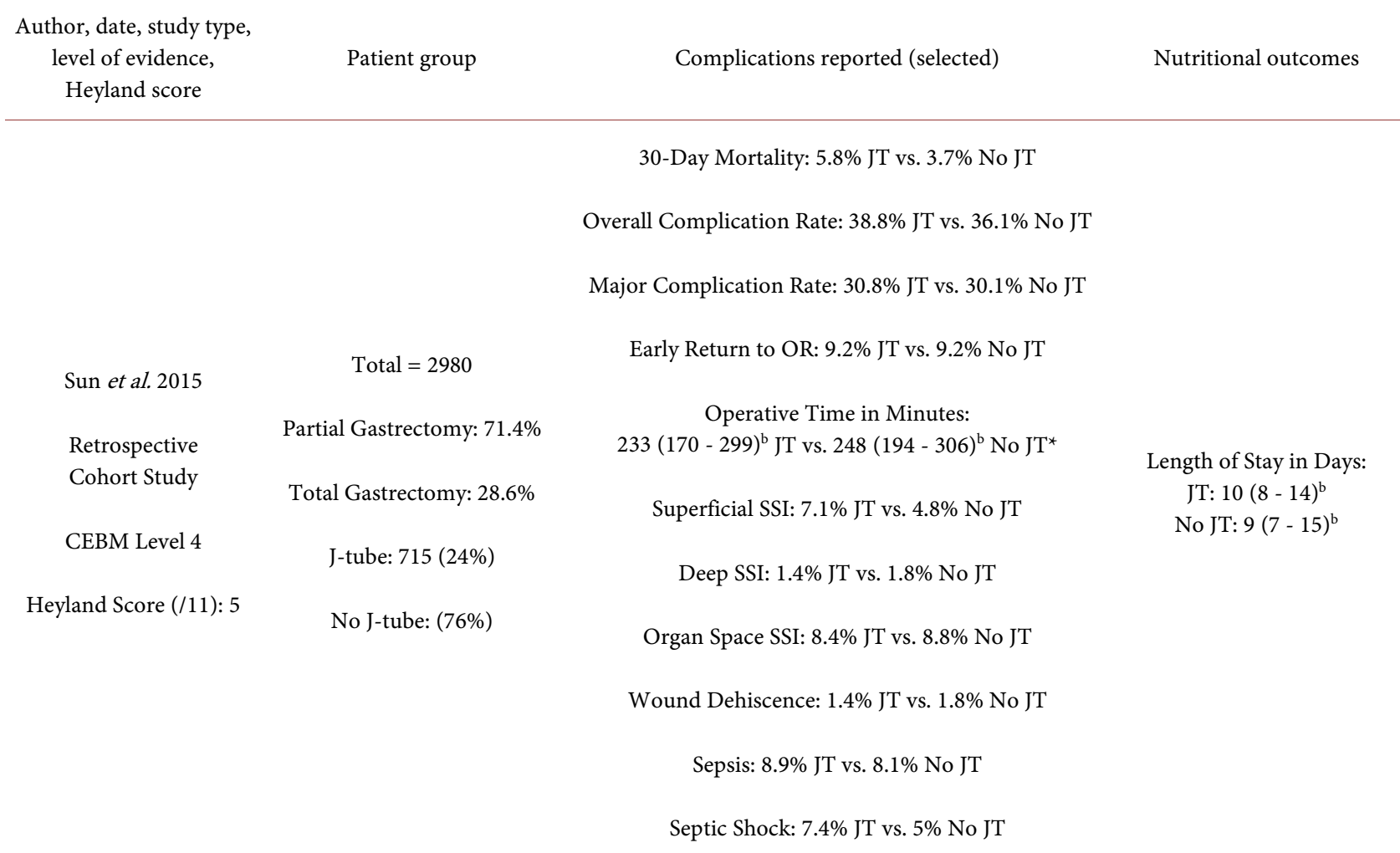

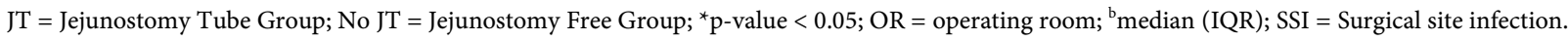


the cause. Nonetheless, the increased rate of UTI is not expected to be directly related to tube insertion and could be managed using standard catheter management practices. Similarly to Dann et al. and Patel et al., time and duration of feed initiation was not discussed, making it difficult to assess the nutritional benefits of jejunostomy tube feeding postoperatively.

\section{Discussion}

Early postoperative feeding improves nutritional outcomes and hence overall morbidity and mortality in gastrointestinal surgery patients [28] [29]. A patient's postoperative nutritional status has also been demonstrated to be of great significance due to its impact on the tolerability of adjuvant therapy following gastric surgery, the delivery of which has been shown to improve patient survival [4]. Adverse effects of chemotherapy such as nausea and vomiting impair a patient's ability to maintain adequate caloric intake solely via oral intake. Enteral nutrition via jejunostomy tube thus offers a practical solution to meet a patient's nutritional needs and maximise their ability to receive chemotherapy with or without radiotherapy postoperatively.

This review assessed the risks and benefits of intraoperative jejunostomy feeding tube insertion and subsequent jejunostomy feeding for patients undergoing total or partial gastrectomy. Four of the five studies were retrospective in nature and therefore, at a high risk of bias. Outcomes reported by the studies were highly heterogenous and aside from the small RCT, many tube-related complications such as dislodgement, blockage, and leakage were not discussed.

Dann et al. and Patel et al. noted increased rates of infectious complications in partial gastrectomy patients who received a jejunostomy feeding tube, and Dann et al. also noted increased rates of any complication, infectious complications, and deep intra-abdominal infection in total gastrectomy patients who received a jejunostomy feeding tube $(\mathrm{p}<0.05)$ [24] [25]. Although jejunostomy and jejunostomy-free groups were found to be highly similar at baseline in both studies, the reasons for selection for jejunostomy tube placement were not described. In addition, the time of initiation and duration of feeding were not mentioned. The study of Wu et al. represents the most likely indicator of the benefits of early enteral nutrition delivered via jejunostomy tube feeding as all jejunostomy patients were fed shortly after gastric resection and continued for 3 months via home enteral nutrition [26]. Notably, jejunostomy fed patients in this study observed lower rates of undernutrition postoperatively and greater postoperative improvements in Karonofsky performance scores when compared with jejunostomy free patients. The data of Sun et al., by far the largest retrospective compilation of gastrectomy patient outcomes, noted no statistically significant differences in 30-day patient mortality, major complication rate, overall complication rate, or infection between jejunostomy and jejunostomy free patients [27]. The small RCT of Heylen et al. noted reduced weight loss in patients who received enteral feeding via jejunostomy tube compared to TPN; however, baseline patient characteristics were not described [11]. 
Due to the lower quality of many of the studies, it was unclear whether standard protocols were used to prevent and manage potentially avoidable complications. Tube-related complications are avoidable with high quality proactive clinical care, including following recommended tube-insertion methods, adequate hygiene and care of the tube and tube site, and consideration of prophylactic antibiotic treatment for infection control which is routinely used in percutaneous endoscopic gastrostomy insertion [30]. Future research is needed to validate protocols for managing preventable complications as discussed above. Overall, this systematic review was limited by the retrospective nature of most of the studies and the high risk of bias associated with them, potential differences in surgical technique, unclear selection criteria for jejunostomy insertion, and unclear or absent description of feed composition and rate of administration.

The findings of this systematic review are currently inconclusive and more stringent prospective research is needed to comprehensively answer the question of whether the jejunostomy feeding tube is the preferred means of postoperative nutritional support for gastrectomy patients and whether or not it should be indicated for all gastrectomy patients or only those at highest risk of postoperative malnutrition due to the risk of tube-related complications. Future studies need to ensure comparable baseline characteristics including disease staging, standardised protocols for jejunostomy feeding tube insertion and care of the tube, feeding regimens, and clear nutrition and tube-related complication outcome measures.

\section{Conclusion}

Overall, this systematic review was limited by the heterogeneity of the studies and their retrospective nature, making it difficult to generalise findings. Studies varied in regards to the complications and nutritional outcomes reported. Other limitations included unclear selection criteria for tube insertion, composition and rate of feeds. Jejunostomy feeding tubes continue to be an inexpensive method of ensuring early enteral nutrition. Jejunostomy feeding tubes may carry a risk of tube related complications, namely tube site infection; and the authors would recommend that these complications be managed proactively if this feeding method is utilised. Home enteral nutrition delivered via jejunostomy feeding tube has been shown to improve postoperative patient nutritional status and quality of life and may improve chemotherapy endurance. With this in mind, future RCTs assessing the risks and benefits of home enteral nutrition via jejunostomy following gastrectomy are recommended.

\section{References}

[1] Parkin, D.M., Bray, F., Ferlay, K. and Pisani, P. (2005) Global Cancer Statistics, 2002. CA: A Cancer Journal for Clinicians, 55, 74-108. https://doi.org/10.3322/canjclin.55.2.74

[2] Australian Institute of Health and Welfare (2014) Cancer in Australia: An Overview 2014. http://www.aihw.gov.au/WorkArea/DownloadAsset.aspx?id=60129550202 
[3] Earle, C. and Mamon, M.D. (2016) Adjuvant and Neoadjuvant Treatment of Gastric Cancer.

http://www.uptodate.com/contents/adjuvant-and-neoadjuvant-treatment-of-gastric -cancer

[4] Cunningham, D., Allum, W.H., Stenning, S.P., Thompson, J.N., Van de Velde, C.J., Nicolson, M., et al. (2006) Perioperative Chemotherapy versus Surgery Alone for Resectable Gastroesophageal Cancer. The New England Journal of Medicine, 355, 11-20. https://doi.org/10.1056/NEJMoa055531

[5] Khomichuk, A.L., Shakhovskaia, A.K., Isakov, V.A., Sharefetdinov, Kh.Kh. and Blokhina, L.V. (2012) Nutritional Status in Patients after Gastrectomy Due to Gastric Cancer. Voprosy Pitaniia, 81, 29-34.

[6] Cidon, E.U. (2010) Nutritional Status after Total Gastrectomy for Gastric Cancer. World Journal of Oncology, 1, 87-90. https://doi.org/10.4021/wjon2010.04.196w

[7] Papini-Berto, S.J. and Burini, R.C. (2001) Causes of Malnutrition in Post-Gastrectomy Patient. Arqivos de Gastroenterologia, 38, 272-275.

[8] Buzby, G.P., Mullen, J.L., Matthews, D.C., Hobbs, C.L. and Rosato, E.F. (1980) Prognostic Nutritional Index in Gastrointestinal Surgery. The American Journal of Surgery, 139, 160-167. https://doi.org/10.1016/0002-9610(80)90246-9

[9] Giner, M., Lavian, A., Meguid, M.M. and Gleason, J.R. (1996) In 1995 a Correlation between Malnutrition and Poor Outcome in Critically Ill Patients Still Exists. Nutrition, 12, 23-29. https://doi.org/10.1016/0899-9007(95)00015-1

[10] Mezhir, J.J., Tang, L.H. and Coit, D.G. (2010) Neoadjuvant Therapy of Locally Advanced Gastric Cancer. Journal of Surgical Oncology, 101, 305-314. https://doi.org/10.1002/jso.21483

[11] Heylen, A.M., Lybeer, M.B., Penninckx, F.M., Kerremans, R.P. and Frost, P.G. (1987) Parenteral versus Needle Jejunostomy Nutrition after Total Gastrectomy. Clinical Nutrition, 6, 131-136. https://doi.org/10.1016/0261-5614(87)90046-X

[12] Baker, A., Wooten, L.A. and Malloy, M. (2011) Nutritional Considerations after Gastrectomy and Esophagectomy for Malignancy. Current Treatment Options in Oncology, 12, 85-95. https://doi.org/10.1007/s11864-010-0134-0

[13] Chen, W., Zhang, Z., Xiong, M., Meng, X., Dai, F., Fang, J., et al. (2014) Early Enteral Nutrition after Total Gastrectomy for Gastric Cancer. Asian Pacific Journal of Clinical Nutrition, 23, 607-611.

[14] Wheble, G.A., Knight, W.R. and Khan, O.A. (2012) Enteral vs. Total Parenteral Nutrition Following Major Upper Gastrointestinal Surgery. International Journal of Surgery, 10, 194-197. https://doi.org/10.1016/j.ijsu.2012.02.015

[15] Sica, G.S., Sujendran, V., Wheeler, J., Soin, B. and Maynard, N. (2005) Needle Catheter Jejunostomy at Esophagectomy for Cancer. Journal of Surgical Oncology, 91, 276-279. https://doi.org/10.1002/jso.20314

[16] Couper, J. (2011) Jejunostomy after Oesophagectomy: A Review of Evidence and Current Practice. The Proceedings of the Nutrition Society, 70, 316-320. https://doi.org/10.1017/S0029665111000553

[17] Rajabi Mashhadi, M.T., Bagheri, R., Ghayour-Mobarhan, M., Zilaee, M., Rezai, R., Maddah, G., et al. (2015) Early Post Operative Enteral versus Parenteral Feeding after Esophageal Cancer Surgery. Iranian Journal of Otorhinolaryngology, 27, 331 336.

[18] Gerndt, S.J. and Orringer, M.B. (1994) Tube Jejunostomy as an Adjunct to Esophagectomy. Surgery, 115, 164-169.

[19] Weimann, A., Braga, M., Harsanyi, L., Laviano, A., Ljungqvist, O., Soeters, P., et al. 
(2006) ESPEN Guidelines on Enteral Nutrition: Surgery including Organ Transplantation. Clinical Nutrition, 25, 224-244. https://doi.org/10.1016/j.clnu.2006.01.015

[20] Lochs, H., Dejong, C., Hammarqvist, F., Hebuterne, X., Leon-Sanz, M., Schütz, T., et al. (2006) ESPEN Guidelines on Enteral Nutrition: Gastroenterology. Clinical Nutrition, 25, 260-274. https://doi.org/10.1016/j.clnu.2006.01.007

[21] Weijs, T.J., Berkelmans, G.H.K., Niewnhuijzen, G.A.P., Ruurda, J.P., Hillegersbeg, R.V., Soeters, P.B., et al. (2015) Routes for Early Enteral Nutrition after Esophagectomy. A Systematic Review. Clinical Nutrition, 34, 1-6. https://doi.org/10.1016/j.clnu.2014.07.011

[22] Heyland, D.K., MacDonald, S., Keefe, M. and Drover, J.W. (1998) Total Parenteral Nutrition in the Critically Ill Patient: A Meta-Analysis. The Journal of the American Medical Association, 280, 2013-2019. https://doi.org/10.1001/jama.280.23.2013

[23] OCEBM Levels of Evidence Working Group (2011) The Oxford Levels of Evidence 2. http://www.cebm.net/wp-content/uploads/2014/06/CEBM-Levels-of-Evidence-2.1.p df

[24] Dann, G.C., Squires, M.H., Postlewait, L.M., Kooby, D.A., Poulstides, G.A., Weber, S.M., et al. (2015) An Assessment of Feeding Jejunostomy Tube Placement at the Time of Resection for Gastric Adenocarcinoma: A Seven-Institution Analysis of 837 Patients from the US Gastric Cancer Collaborative. Journal of Surgical Oncology, 112, 195-202. https://doi.org/10.1002/jso.23983

[25] Patel, S.H., Kooby, D.A., Staley, C.A. and Maithel, S.K. (2013) An Assessment of Feeding Jejunostomy Tube Placement at the Time of Resection for Gastric Adenocarcinoma. Journal of Surgical Oncology, 107, 728-734. https://doi.org/10.1002/jso.23324

[26] Wu, Q., Yu, J.C., Kang, W.M. and Ma, Z.Q. (2011) Short-Term Effects of Supplementary Feeding with Enteral Nutrition via Jejunostomy Catheter on Post-Gastrectomy Gastric Cancer Patients. Chinese Medical Journal, 124, 97-301.

[27] Sun, Z., Shenoi, M.M., Nussbaum, D.P., Keenan, J.E., Gulack, B.C., Tyler, D.S., et al. (2016) Feeding Jejunostomy Tube Placement during Resection of Gastric Cancers. Journal of Surgical Research, 200, 189-194. https://doi.org/10.1016/j.jss.2015.07.014

[28] Vaithiswaran, V., Srinivasan, K. and Kadambari, D. (2008) Effect of Early Enteral Feeding after Upper Gastrointestinal Surgery. Tropical Gastroenterology, 29, 91-94.

[29] Osland, E.J. and Memon, M.A. (2010) Early Postoperative Feeding in Resectional Gastrointestinal Surgical Cancer Patients. World Journal of Gastrointestal Oncology, 2, 187-191. https://doi.org/10.4251/wjgo.v2.i4.187

[30] Blumenstein, I., Shastri, Y.M. and Stein, J. (2014) Gastroenteric Tube Feeding: Techniques, Problems and Solutions. World Journal of Gastroenterology, 20, 85058524. https://doi.org/10.3748/wjg.v20.i26.8505 
Submit or recommend next manuscript to SCIRP and we will provide best service for you:

Accepting pre-submission inquiries through Email, Facebook, LinkedIn, Twitter, etc. A wide selection of journals (inclusive of 9 subjects, more than 200 journals)

Providing 24-hour high-quality service

User-friendly online submission system

Fair and swift peer-review system

Efficient typesetting and proofreading procedure

Display of the result of downloads and visits, as well as the number of cited articles Maximum dissemination of your research work

Submit your manuscript at: http://papersubmission.scirp.org/

Or contact ojgas@scirp.org 